\title{
Deactivation of metastable single-crystal silicon hyperdoped with sulfur
}

\section{Citation}

Simmons, C. B., Austin J. Akey, Jacob J. Krich, Joseph T. Sullivan, Daniel Recht, Michael J. Aziz, and Tonio Buonassisi. 2013. "Deactivation of Metastable Single-Crystal Silicon Hyperdoped with Sulfur." Journal of Applied Physics 114 (24) (December 28): 243514. doi:10.1063/1.4854835.

\section{Published Version}

doi:10.1063/1.4854835

\section{Permanent link}

http://nrs.harvard.edu/urn-3:HUL.InstRepos:14550026

\section{Terms of Use}

This article was downloaded from Harvard University's DASH repository, and is made available under the terms and conditions applicable to Other Posted Material, as set forth at http:// nrs.harvard.edu/urn-3:HUL.InstRepos:dash.current.terms-of-use\#LAA

\section{Share Your Story}

The Harvard community has made this article openly available.

Please share how this access benefits you. Submit a story.

\section{Accessibility}




\title{
Deactivation of metastable single-crystal silicon hyperdoped with sulfur
}

\author{
C. B. Simmons, ${ }^{1, a)}$ Austin J. Akey, ${ }^{1}$ Jacob J. Krich, ${ }^{2}$ Joseph T. Sullivan, ${ }^{1}$ Daniel Recht, ${ }^{3}$ \\ Michael J. Aziz, ${ }^{3}$ and Tonio Buonassisi ${ }^{1}$ \\ ${ }^{1}$ Massachusetts Institute of Technology, Cambridge, Massachusetts 02139, USA \\ ${ }^{2}$ University of Ottawa, Ottawa, Ontario K1N 6N5, Canada \\ ${ }^{3}$ Harvard School of Engineering and Applied Sciences, Cambridge, Massachusetts 02138, USA
}

(Received 1 October 2013; accepted 9 December 2013; published online 30 December 2013)

\begin{abstract}
Silicon supersaturated with sulfur by ion implantation and pulsed laser melting exhibits broadband optical absorption of photons with energies less than silicon's band gap. However, this metastable, hyperdoped material loses its ability to absorb sub-band gap light after subsequent thermal treatment. We explore this deactivation process through optical absorption and electronic transport measurements of sulfur-hyperdoped silicon subject to anneals at a range of durations and temperatures. The deactivation process is well described by the Johnson-Mehl-AvramiKolmogorov framework for the diffusion-mediated transformation of a metastable supersaturated solid solution, and we find that this transformation is characterized by an apparent activation energy of $E_{A}=1.7 \pm 0.1 \mathrm{eV}$. Using this activation energy, the evolution of the optical and electronic properties for all anneal duration-temperature combinations collapse onto distinct curves as a function of the extent of reaction. We provide a mechanistic interpretation of this deactivation based on short-range thermally activated atomic movements of the dopants to form sulfur complexes. (C) 2013 AIP Publishing LLC. [http://dx.doi.org/10.1063/1.4854835]
\end{abstract}

\section{INTRODUCTION}

Using ion implantation followed by nanosecond pulsed laser melting (PLM), it is possible to dope silicon with impurity elements to concentrations that are orders of magnitude above the equilibrium solid-solubility limit while maintaining a single-crystal, single-phase solid solution. ${ }^{1,2}$ Such hyperdoped silicon is of interest for a variety of potential applications. The enhanced conductivity of silicon hyperdoped with shallow-level dopants ( $\mathrm{B}, \mathrm{Sb}, \mathrm{P}, \mathrm{As}$ ) has been studied for potential use in low-resistivity junctions for integrated circuits. ${ }^{3-5}$ Silicon hyperdoped with deep-level dopants (chalcogens, transition metals) has optoelectronic applications (photovoltaics, photodetectors, light emitters) ${ }^{6-9}$ due to its enhanced broadband infrared absorption ${ }^{10-12}$ and extended infrared photoresponse. . $^{6,13}$

Hyperdoped silicon is a metastable, supersaturated solid solution. As such, its enhanced properties-increased conductivity in the case of shallow dopants and sub-band gap optical absorption in the case of deep-level dopants - deactivate upon subsequent thermal treatment. ${ }^{4,10,14-23}$ There has been much interest in studying the nature of this deactivation. Studies on silicon hyperdoped with shallow dopants have correlated dopant deactivation (i.e., reductions in conductivity) with the formation of inactive dopant clusters or precipitates, depending upon the dopant element. ${ }^{17,20,21}$ Studies on the deactivation of sub-band gap absorptance in silicon made polycrystalline and hyperdoped with chalcogens by femtosecond laser irradiation attributed the deactivation to long-range dopant diffusion to and precipitation on grain boundaries. ${ }^{22,24}$

In this work, we examine the annealing-induced deactivation of single-crystal, sulfur-hyperdoped silicon. We perform

\footnotetext{
a) Author to whom correspondence should be addressed. Electronic mail: christie.simmons@gmail.com
}

optical absorption, van der Pauw, and Hall measurements to probe the chemical state of the sulfur dopants, and we perform Secondary Ion Mass Spectrometry (SIMS) and cross-sectional transmission electron microscopy (XTEM) on a heavily annealed sample to probe the location and distribution of the dopants in the deactivated state. From these measurements, we characterize the apparent deactivation energy and gain insight into the underlying physical transformation caused by thermal treatment of this metastable material.

\section{MATERIALS AND METHODS}

Double-side polished p-type $\operatorname{Si}(001)$ wafers $778 \mu \mathrm{m}$ thick (boron doped, $\rho=10-30 \Omega \mathrm{cm}$ ) were commercially ion implanted with ${ }^{32} \mathrm{~S}^{+}$at $95 \mathrm{keV}$ to a dose of $1 \times 10^{16} \mathrm{~cm}^{-2}$. The implant was performed at room temperature, with the silicon wafer tilted $7^{\circ}$ relative to the incident beam to minimize ion channeling. The ion implantation process resulted in an amorphous surface layer containing most of the implanted sulfur with a concentration peak approximately $120 \mathrm{~nm}$ deep. Implanted samples were irradiated with four pulses from a spatially homogenized, pulsed $\mathrm{XeCl}^{+}$excimer laser $(\lambda=308 \mathrm{~nm}, 25 \mathrm{~ns}$ duration full width at half maximum, square spot size approximately $3 \times 3 \mathrm{~mm}^{2}$ ) with fluences of $1.7 \mathrm{~J} / \mathrm{cm}^{2}$ for the first three pulses and $1.8 \mathrm{~J} / \mathrm{cm}^{2}$ for the final pulse. The laser pulses melted the sample surface to a depth of approximately $450 \mathrm{~nm}$, deeper than the extent of implantation-induced amorphization. The melted layer then resolidified epitaxially from the underlying, un-melted silicon substrate. The melt depth was deduced from in situ time-resolved reflectivity measurements of the melt duration and heat flow simulations of the melting and solidification. ${ }^{25}$ The resolidified material is single crystalline, free of extended defects, and doped with sulfur to a concentration of almost $1 \%$ atomic. $^{10,11,25}$ 
The samples were RCA cleaned to remove organic and metallic contaminants and were then individually subjected to thermal annealing in a Jipelec Jetfirst Rapid Thermal Processor (RTP). We performed 27 different anneals covering a range of temperatures $(500 \mathrm{~K}, 550 \mathrm{~K}, 600 \mathrm{~K}, 700 \mathrm{~K}, 800 \mathrm{~K}$, $900 \mathrm{~K}, 1000 \mathrm{~K}, 1100 \mathrm{~K})$ and durations $(10 \mathrm{~s}, 32 \mathrm{~s}, 100 \mathrm{~s}, 316 \mathrm{~s}$, $1000 \mathrm{~s}, 3162 \mathrm{~s})$. All samples were annealed in an Ar atmosphere while resting upon a cleaned silicon wafer. For all anneals, the temperature rise was set at $75^{\circ} / \mathrm{s}$ and the cool down rate was as fast as was possible without removing samples from the RTP. Practically, cooling was limited by radiation from the sample to on the order of $10^{\circ} / \mathrm{s}$. Unless otherwise stated, the anneal temperatures, $T$, and durations, $t$, listed for each anneal condition correspond to the set points of the RTP for the plateau (i.e., they do not account for the ramp up and ramp down, or any temperature offsets). A thermocouple in contact with the back of the silicon wafer recorded the actual temperature-time profile for each anneal, $T_{M}(t)$. The temperature measured by the thermocouple varied from the set temperature by up to $\sim 8 \mathrm{~K}$. The temperature-time profiles measured by the thermocouple including the ramp up to the set point plateau and the cool down to room temperature are used to calculate an effective anneal duration, $t_{\text {eff }}$, discussed in Sec. III A and defined in Eq. (5). A control sample which received the same ion implantation and pulse laser melting but no subsequent thermal anneal is included for reference and is labeled as "No Anneal."

The optical absorption from $700-2300 \mathrm{~nm}$ was measured for each sample both before and after thermal annealing. The transmission $(T)$ and reflection $(R)$ were measured using a PerkinElmer Lambda 950 UV/Vis/NIR Spectrometer equipped with an integrating sphere, and the absorptance, $A$, was calculated according to $A=(1-T-R) /(1-R)$. A silver mirror was used as a $100 \%$ reflectance standard, and the illumination area of the spectrometer was apertured to ensure that the measurement probed only the laser melted region of the sample.

After the thermal anneals and absorption measurements, all samples were fabricated into van der Pauw devices. Following the procedure in Ref. 26, photolithography and $\mathrm{SF}_{6}$ reactive ion etching to a depth of $2 \mu \mathrm{m}$ were used to to define a cloverleaf structure and to electrically isolate the crystalline, laser melted region of interest from the un-melted, amorphized surface region. The outer and inner diameters of the cloverleaf structure were $2 \mathrm{~mm}$ and $0.2 \mathrm{~mm}$, respectively, and four metal contacts $100 \mu \mathrm{m}$ in diameter were deposited on the outer edges $(20 \mathrm{~nm} \mathrm{Ti}, 20 \mathrm{~nm} \mathrm{Ni}$, $200 \mathrm{~nm} \mathrm{Ag} \mathrm{stack).} \mathrm{All} \mathrm{measurements} \mathrm{were} \mathrm{performed} \mathrm{at}$ room temperature and Hall measurements were acquired to a maximum magnetic field of $1.5 \mathrm{~T}$.

SIMS was performed to compare the sulfur concentration profiles in an annealed and a non-annealed sample using a Physical Electronics 6650 Dynamic SIMS instrument. Measurements were performed using a $6 \mathrm{keV}$ Cs ion beam at $1 \mathrm{nA}$. SIMS craters were $50 \mu \mathrm{m}$ square, with depth calibration determined ex-situ by contact profilometry. Absolute concentrations of the ${ }^{32} \mathrm{~S}$ isotope of sulfur were calibrated against known ion-implantation doses from the as-implanted regions of each sample, normalized by the ${ }^{28} \mathrm{Si}$ signal.

\section{RESULTS AND ANALYSIS}

\section{A. Deactivation of the sub-band gap optical absorption}

Figure 1 shows the optical absorptance for a selection of samples subject to varying annealing conditions. The baseline absorptance of the sample that was not annealed ("No Anneal") and the absorptance of the plain silicon substrate without the hyperdoped sulfur layer ("Si Substrate") are also shown for reference. Consistent with previous literature, the un-annealed sample exhibits strong, broadband absorption at photon energies below the silicon band gap $(1.1 \mathrm{eV}$ or $1100 \mathrm{~nm})$ while the reference silicon substrate has no such absorption. This enhanced sub-band gap optical absorption exhibited by the sulfur-hyperdoped silicon is deactivated by subsequent thermal anneals. Fig. 1 shows that the sub-band gap absorptance decreases monotonically with anneal temperature (a) or anneal duration (b). For anneal temperatures above $800 \mathrm{~K}$, the sub-band gap absorptance is essentially indistinguishable from the reference silicon substrate.
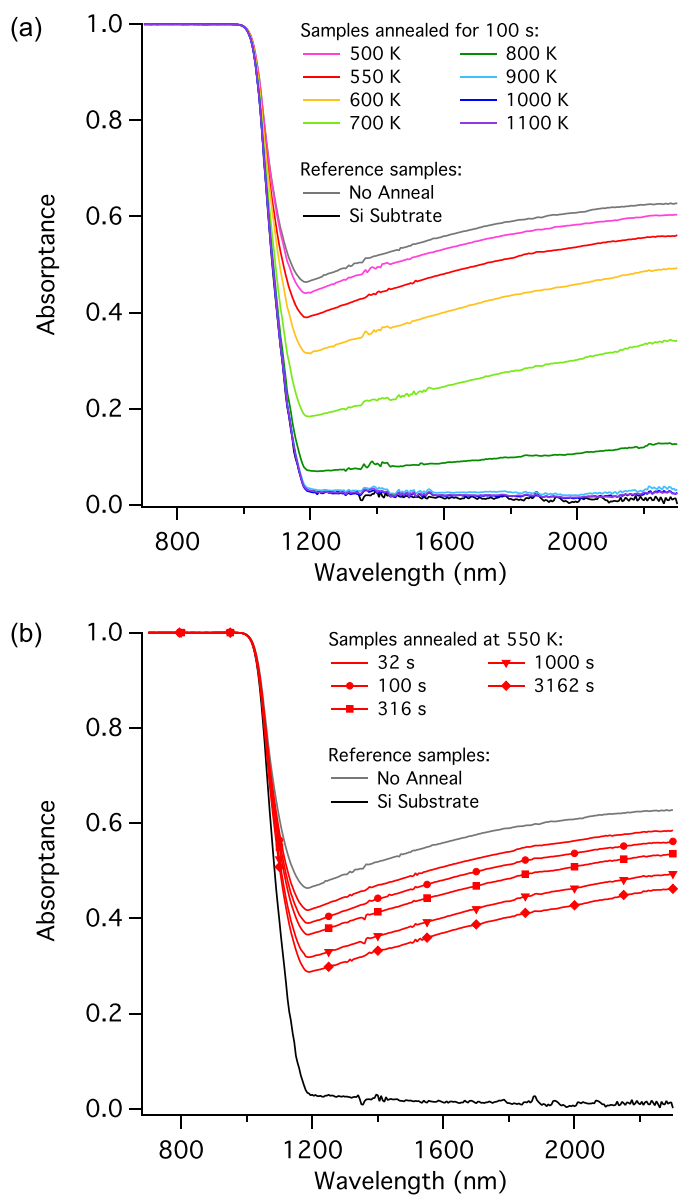

FIG. 1. Optical absorptance of silicon hyperdoped with sulfur. (a) Absorptance for samples annealed for $100 \mathrm{~s}$ at the temperatures indicated in the legend. (b) Absorptance for samples annealed at $550 \mathrm{~K}$ for durations indicated in the legend. The reference absorptance of an un-annealed sample and the plain silicon substrate are also plotted in (a) and (b). The slight nonzero sub-band gap absorptance in the reference silicon substrate is due to the noise floor of the measurement and a small offset resulting from the use of the silver mirror, which does not have perfect $100 \%$ reflection in this wavelength range, for a reflectance standard. 
It has previously been shown that the sub-band gap absorptance scales with the sulfur concentration in the hyperdoped layer, with no significant absorptance for concentrations below approximately $1.4 \times 10^{19} \mathrm{~cm}^{-3} \cdot{ }^{11,23,27}$ Thus, one might imagine that the decrease in sub-band gap absorptance with annealing is caused by sulfur in the hyperdoped layer either evaporating from the surface or diffusing deeper into the silicon wafer, resulting in a lower peak sulfur concentration. However, the SIMS data presented in Fig. 2(a) contradict this explanation.

Figure 2(a) shows that the sulfur concentration profiles for the un-annealed sample and the sample that was annealed at $1100 \mathrm{~K}$ for $100 \mathrm{~s}$ are nearly identical. However, despite the similarity of the sulfur distribution, Fig. 1(a) shows that the un-annealed sample exhibits strong sub-band gap absorptance while the sample annealed at $1100 \mathrm{~K}$ for $100 \mathrm{~s}$ has no measurable sub-band gap absorptance. Therefore, we hypothesize that the reduction in sub-band gap absorptance results from a change in the chemical state of the sulfur dopants rather than long-range dopant diffusion.

An alternative explanation for the deactivation of subband gap absorptance could be precipitation of sulfur out of the supersaturated solution into a second phase. We performed XTEM on the most heavily annealed sample (anneal conditions: $1100 \mathrm{~K}$ for $100 \mathrm{~s}$ ) to attempt to identify such sulfur precipitates. The results, shown in Fig. 2(b), indicate that if such precipitates exist, they are either smaller than the

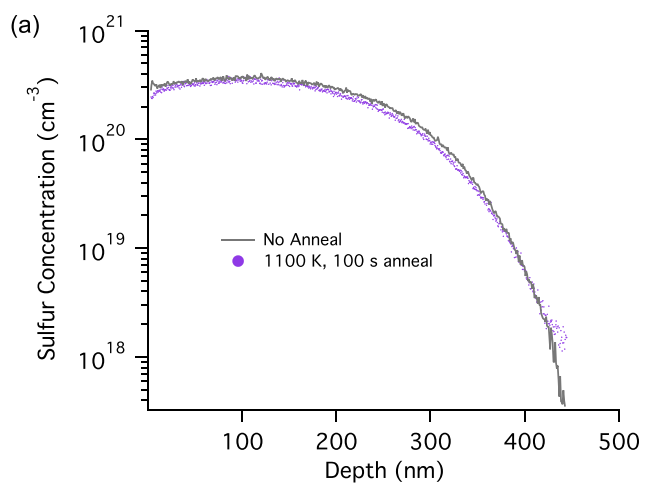

(b)

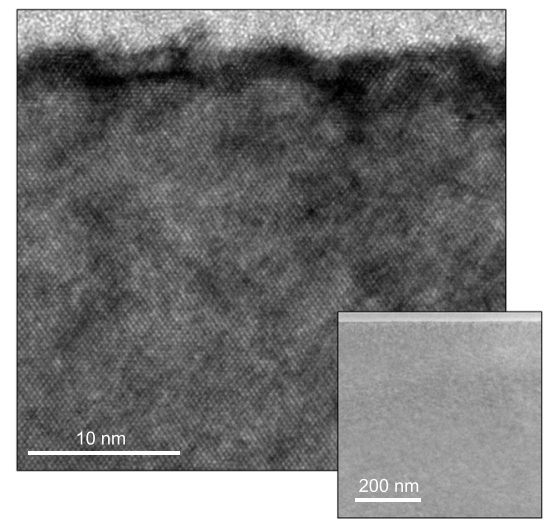

FIG. 2. (a) SIMS data of the sulfur concentration vs. depth for a sample that was not annealed (gray line) and for the sample annealed at $1100 \mathrm{~K}$ for $100 \mathrm{~s}$ (purple dots). The absorptance of the annealed sample is shown by the purple trace in Fig. 1(a). (b) XTEM of the annealed sample in (a) (anneal conditions: $1100 \mathrm{~K}$ for $100 \mathrm{~s}$ ). Inset: a zoomed-out image showing a larger sample area. Both images show the surface of the sample, the protective carbon film on the surface, and the underlying single-crystal hyperdoped silicon. instrument can resolve by bright-field contrast (on the order of $5 \mathrm{~nm}$ for sulfur in silicon) or coherently lattice matched with silicon, because they produce no detectable diffraction contrast, no interruption to the visible lattice fringes, no Moiré fringes, etc. Such lattice-matched precipitates may be present and undetectable because there may not be sufficient scattering contrast between sulfur and silicon to distinguish very small second-phase particles in bright-field XTEM. Analogous undetectable, so called "ninja" precipitates are believed to exist for oxygen in silicon. ${ }^{28}$

For the remainder of the manuscript, we explore the kinetics of deactivation under thermal annealing, and we provide a mechanistic interpretation for the atomic scale transformation of sulfur-hyperdoped silicon that can explain the evolution of the material's optical and electronic properties.

\section{B. Evolution of optical absorption and electronic transport properties with annealing}

We examine the deactivation kinetics by analyzing the evolution of the sub-band gap absorptance as a function of both anneal temperature and duration. We quantify the subband gap absorptance for each sample by averaging the absorptance from $1200-2300 \mathrm{~nm}, \bar{A}$. The results for all 27 anneal conditions and the un-annealed reference sample are shown in Fig. 3(a), color coded by anneal temperature and plotted as a function of the anneal duration. The average absorptance for all of the samples after pulsed laser melting but prior to thermal annealing is $\bar{A}=0.571 \pm 0.004$. The uncertainty is the standard deviation of the measurements, which takes into account both measurement repeatability and any sample-to-sample variation. This un-annealed value of $\bar{A}$ is the black data point in Fig. 3(a) with a corresponding anneal duration of $0 \mathrm{~s}$. The uncertainty in $\bar{A}$ for the annealed absorptance curves is calculated from the relative uncertainty for the separate reflectance and transmission measurements, determined from the repeated measurements on the unannealed samples.

We gain further insight into the transformation of the chemical state of the sulfur dopants by probing the materials' electronic properties through van der Pauw and Hall measurements. Figs. 3(b) and 3(c) show the measured electron mobility, $\mu_{e}$, and sheet carrier density, $n_{\mathrm{s}}$, for each of the samples in (a) also plotted as functions of the anneal duration and using the same color coding for the anneal temperature. Because the dopant concentration in hyperdoped layer is non-uniform (see Fig. 2(a)), $n_{\mathrm{s}}$ and $\mu_{e}$ represent effective quantities, or weighted averages of the depth-dependent carrier concentration and mobility. ${ }^{29}$ The error bars in (b) and (c) are determined by the finite size and placement of the contacts on the cloverleaf van der Pauw structure according to Ref. 30.

The qualitative trends of $\bar{A}$ with anneal temperature and anneal duration in Fig. 3(a) show a monotonic decrease, as was discussed in reference to Fig. 1. Figs. 3(b) and 3(c) show that $\mu_{e}$ and $n_{\mathrm{s}}$ also evolve with anneal temperature and duration, but the trends are qualitatively different. For the lower temperature anneals, $<600 \mathrm{~K}, \mu_{e}$ remains essentially 


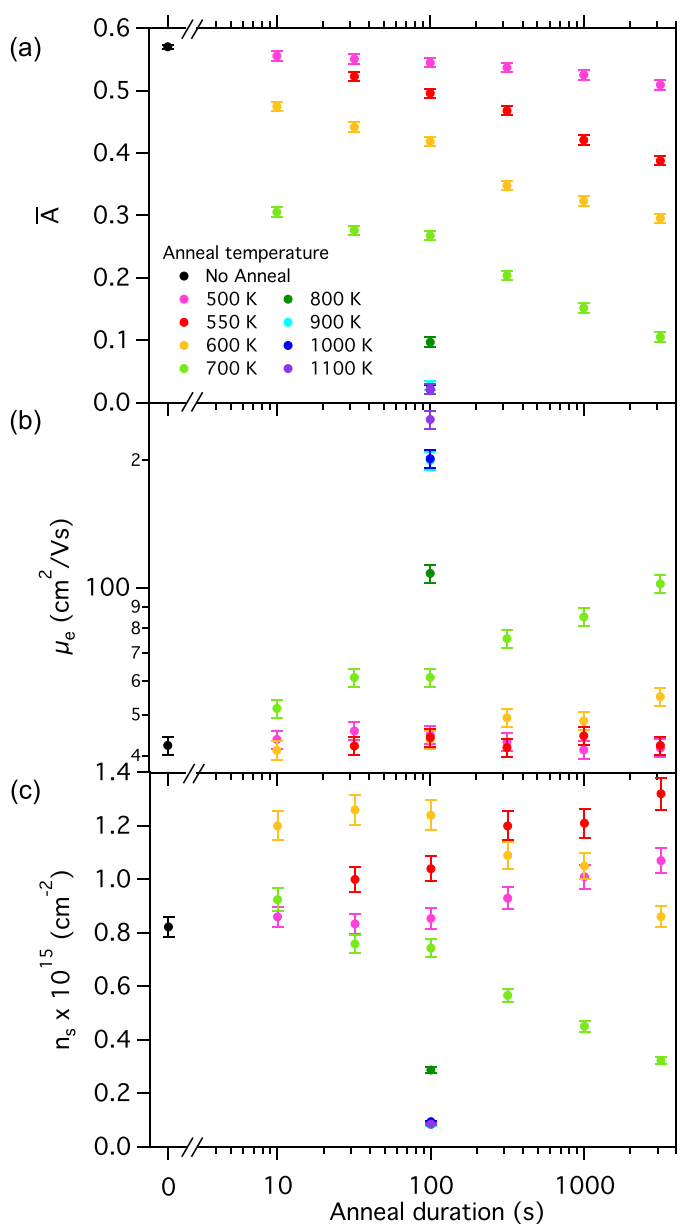

FIG. 3. Evolution of the optical and electrical properties with annealing duration and temperature. (a) Average sub-band gap absorptance from 1200-2300 nm. ((b) and (c)) Electron mobility and sheet carrier concentration, respectively, for each of the samples in (a). In all panels, the black data points indicate the corresponding measurements on the un-annealed sample (anneal duration of zero).

unchanged. Only for higher temperature anneals does it show a marked increase. $n_{\mathrm{s}}$, meanwhile, increases with anneal duration for temperatures $<600 \mathrm{~K}$, decreases with anneal duration for temperatures $>600 \mathrm{~K}$, and is nonmonotonic (first increasing then decreasing) for the anneals at $600 \mathrm{~K}$. This remarkably non-monotonic evolution of $n_{\mathrm{s}}$ serves as motivation for the 3 -state mechanistic interpretation advanced in Sec. IV. In Sec. IIIC, we determine the apparent activation energy associated with the deactivation of the optical absorptance, and we show that this single activation energy can be used to evaluate all three data sets in Fig. $3\left(\bar{A}, \mu_{e}\right.$, and $\left.n_{\mathrm{s}}\right)$.

\section{Deactivation kinetics}

Sulfur-hyperdoped silicon is a supersaturated solid in which the sulfur dopant is trapped in solution at concentrations several orders of magnitude higher than the equilibrium solubility limit as a result of the rapid solidification following pulsed laser melting. The deactivation upon subsequent thermal treatment reported in Sec. III B is consistent with the classical kinetics of state transformations. The JohnsonMehl-Avrami-Kolmogorov (JMAK) equation is a general framework to describe diffusion-mediated changes of state in metastable solid solutions. ${ }^{31,32}$ Following the procedure of Sec. 9.6.15 in Ref. 33, we determine the apparent activation energy for this decomposition using the JMAK equation applied to a set of isothermal anneals performed at various temperatures. It is not necessary to have a specific mechanism and kinetic model to perform this quantitative analysis. Further discussion of the potential mechanism in this material is presented in Sec. IV.

Equation (1) is the JMAK equation; it describes the progress of a change in state in terms of the fraction transformed, $f$

$$
f=1-\exp \left(-k t^{n}\right)
$$

where $t$ is the time duration, $n$ is a constant, and $k$ is a temperature dependent rate constant. We use the average subband gap absorptance, $\bar{A}$, to calculate the fraction transformed following Eq. (9.20) in Ref. 33:

$$
f=\frac{\bar{A}-\bar{A}_{\mathrm{NA}}}{\bar{A}_{\mathrm{Si}}-\bar{A}_{\mathrm{NA}}},
$$

where $\bar{A}_{\mathrm{NA}}$ is the average sub-band gap absorptance of the un-annealed hyperdoped silicon (the initial, optically active value prior to transformation) and $\bar{A}_{\mathrm{Si}}$ is the absorptance of the reference silicon substrate (the final, optically inactive value at the end of the transformation). By rewriting Eq. (1) into the form

$$
\ln (-\ln (1-f))=\ln k+n \ln (t),
$$

we see that for a constant anneal temperature, a plot of $\ln (-\ln (1-f))$ vs. $\ln (t)$ should give a straight line. Such a plot is shown in Fig. 4(a) along with fits using Eq. (3) for the samples annealed at $500 \mathrm{~K}, 550 \mathrm{~K}, 600 \mathrm{~K}$, and $700 \mathrm{~K}$. We extract $\tau$, the time constant for $63 \%$ transformation $(f=1-1 / e)$ at each anneal temperature from the fits. Fig. 4(b) shows an Arrhenius plot of $\tau$ determined for each of the curves in (a), and the fit shown gives an energy $E_{A}=1.7 \pm 0.1 \mathrm{eV}$ for the deactivation of the sub-band gap optical absorption in sulfur-hyperdoped silicon.

Both higher annealing temperature and longer annealing duration increase the extent of the transformation of the sulfur dopant from the optically active to the optically inactive chemical state. The apparent activation energy, $E_{A}$ determines the trade off between the anneal temperature and duration. Thus, for a given set of annealing conditions we can define a single parameter, $Q$, which characterizes the extent of the reaction:

$$
Q=t_{\text {eff }} \times \exp \left(-E_{A} / k_{B} T\right),
$$

where $k_{B}$ is Boltzmann constant, and we have introduced $t_{\mathrm{eff}}$, the effective annealing duration. $t_{\mathrm{eff}}$ is determined by the actual temperature-time profile of the thermal anneals (as measured by the thermocouple in the RTP), and it takes into account the effect of dynamics that occur during the heating and cooling and any offset from the set temperature: ${ }^{34}$ 
(a)
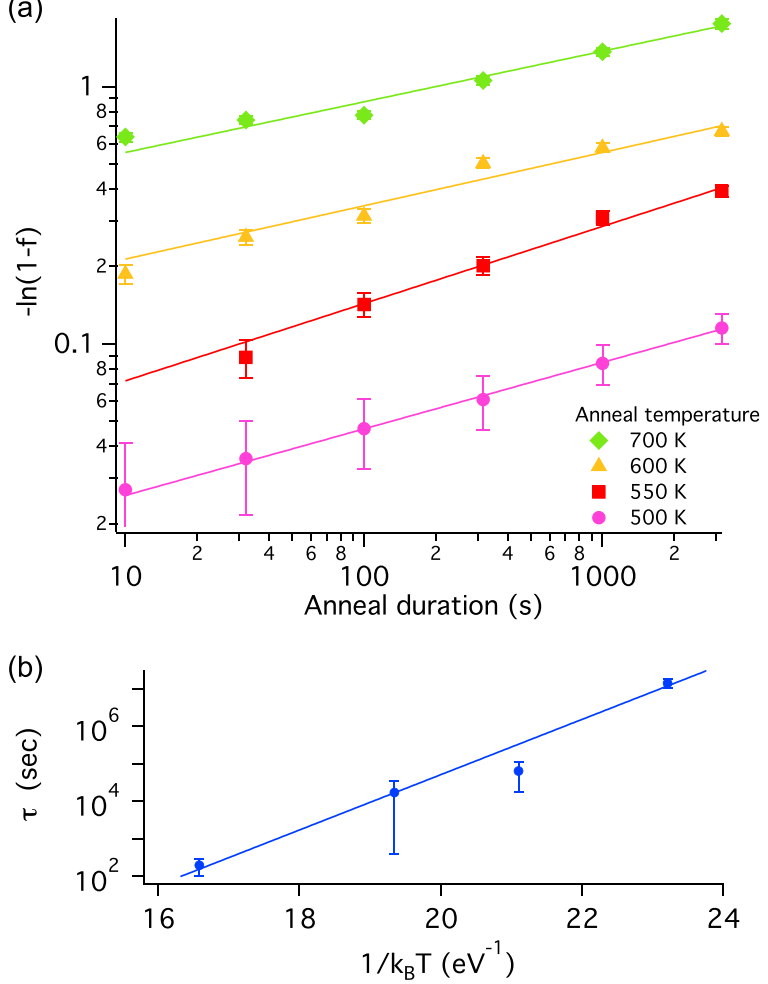

FIG. 4. (a) The average sub-band gap absorptance $(1200-2300 \mathrm{~nm})$ of samples annealed for various durations at $500 \mathrm{~K}, 550 \mathrm{~K}, 600 \mathrm{~K}$, and $700 \mathrm{~K}$, normalized as the fraction transformed, $f$, by Eq. (2). The error bars were propagated from uncertainty in the measurements of $\bar{A}$ (b) An Arrhenius plot of $\tau$, the time constant corresponding to $63 \%$ transformation, as a function of the anneal temperature. The error bars were determined by the quality of the fits in (a).

$$
t_{\mathrm{eff}}=\int \exp \left[E_{A} / k_{B} T-E_{A} / k_{B} T_{M}(t)\right] d t
$$

where $T$ is the set point temperature, $E_{A}$ is the activation energy determined above, $T_{M}(t)$ is the thermocouple measured temperature-time profile, and the integration is calculated over the entire heating cycle for which $T_{M}>305 \mathrm{~K}$. Using Eqs. (4) and (5) we calculate the extent of reaction for each of the 27 different anneal conditions. Fig. 5(a) plots $\bar{A}$ as a function of the normalized extent of reaction, $\tilde{Q}=Q / Q_{0}$, where $Q_{0}$ is the smallest extent of reaction measured (and corresponds to the shortest and lowest temperature anneal conditions).

As expected, Fig. 5(a) shows that samples annealed at different temperature/duration conditions, but yielding a similar extent of reaction, have correspondingly similar $\bar{A}$. In other words, all of the data points from Fig. 3(a) collapse onto a single curve. We conclude from this result that the transformation of sulfur-hyperdoped silicon to an optically inactive state upon annealing is well described by the single apparent activation energy, $E_{A}$.

The same activation energy, $E_{A}$ is used to re-plot the data in Figs. 3(b) and 3(c) along the extent of reaction axis in Figs. 5(b) and 5(c). Two things are immediately apparent from Fig. 5. First, similar to $\bar{A}$, the values of $\mu_{e}$ and $n_{\mathrm{s}}$ for all samples annealed with different temperature-duration conditions collapse onto single curves determined by the extent of

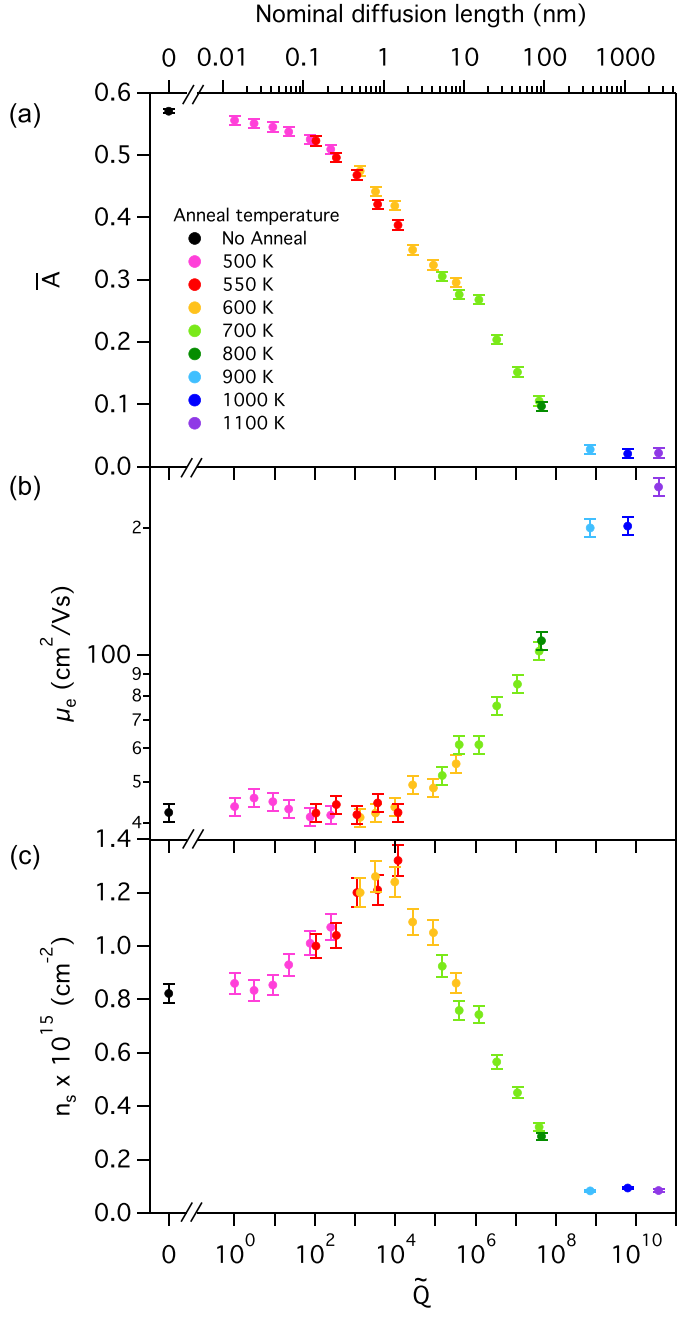

FIG. 5. Evolution of the optical and electrical properties with annealing, transformed onto an axis characterizing the extent of the reaction, $\tilde{Q}$, (bottom) or the nominal diffusion length (top). Panels (a)-(c) show the same data as (a)-(c) in Fig. 3, with the same vertical scales. The anneal conditions for each sample (temperature and duration) have been transformed using the experimentally determined apparent activation energy, $E_{A}=1.7 \pm 0.1 \mathrm{eV}$, and Eq. (4) (bottom axis) or Eq. (6) (top axis).

reaction, $Q$. Therefore, the evolution of the electronic properties provides additional information about the transformation of the sulfur dopant. Second, the qualitative trends of $\mu_{e}$ and $n_{\mathrm{s}}$ are very different. The mobility has no significant change until $\bar{A}$ has decayed to about $61 \%$ of its initial value $(\bar{A} \approx 0.35)$, and $n_{\mathrm{s}}$ has a distinctive non-monotonic trend. In agreement with Ref. 35, the opposing trends of $\bar{A}$ and $n_{\mathrm{s}}$ are further evidence that the enhanced sub-band gap absorptance exhibited by chalcogen hyperdoped silicon is not due to free carrier absorption, but rather due to optical transitions involving the intra-gap impurity states.

Section IV provides further analysis and interpretation of the data presented in Fig. 5, including a potential mechanistic interpretation of the deactivation process.

\section{DISCUSSION}

\section{A. The role of diffusion}

In Ref. 22 the deactivation of sub-band gap absorptance in polycrystalline chalcogen hyperdoped silicon was attributed 
to the diffusion of the dopant to grain boundaries, where it formed optically inactive precipitates. The sulfur-hyperdoped silicon material studied here is a supersaturated solid solution, and similar to the polycrystalline case, one possible explanation of the deactivation of the sub-band gap absorptance is a diffusional transformation of the sulfur dopants into optically inactive defect clusters or precipitates. However, in contrast to the polycrystalline case, the material studied here is singlecrystalline and free of any extended defects. Despite this important difference, deactivation of sub-band gap absorptance is qualitatively similar for these two materials.

Even without the presence of grain boundaries, deactivation may be caused by local rearrangement of sulfur atoms into optically inactive clusters by atomic diffusion. The activation energy for the diffusion of sulfur in silicon has been previously reported as $E_{D}=1.8 \mathrm{eV}$, and has been associated with diffusion via a kick-out mechanism. ${ }^{36,37}$ This value is slightly higher than, but within the uncertainty of, the apparent activation energy measured here. This literature value was determined in the case of dilute concentrations of sulfur diffused into silicon from a vapor source. In contrast, we have extremely high sulfur concentrations that could result in a lower apparent activation energy. As is discussed in Sec. IV B, the evolution of the optical and electronic properties with annealing reported here could be explained by the dimerization of the sulfur dopants, and a lower apparent activation energy for short-range diffusion involving dimerization than for long-range diffusion is quite plausible.

Using the diffusion coefficient $D_{0}=0.047 \mathrm{~cm}^{2} / \mathrm{s}$ measured for dilute concentrations of sulfur in silicon, ${ }^{36}$ we can rescale the extent of reaction axis into a nominal diffusion length according to

$$
d=\sqrt{D_{0} \times t_{\text {eff }} \times \exp \left(-E_{A} / k_{B} T\right)}=\sqrt{D_{0} * Q} .
$$

This rescaling, presented as the top axis in Fig. 5, represents only a nominal diffusion length because we have combined the literature diffusion coefficient with the apparent activation energy determined above. For the sample annealed at $1100 \mathrm{~K}$, the nominal diffusion length is $2.6 \mu \mathrm{m}$, while the diffusion length calculated using $D_{0}$ and $E_{D}$ from Ref. 36 is $1.6 \mu \mathrm{m}$. The SIMS data for this sample, however, does not show transport of sulfur over these length scales (Fig. 2(a)). This lack of long-range sulfur diffusion in annealed, single-crystal, sulfur-hyperdoped silicon has been reported previously, ${ }^{10}$ and could be explained by the formation of stable sulfur defect clusters that inhibit further diffusion.

\section{B. Evolution of the sulfur chemical state}

Here, we provide a mechanistic interpretation of the evolution of the sulfur chemical state upon annealing of hyperdoped silicon that is consistent with the observed trends in the material's optical and electronic properties presented in Fig. 5.

The simplest possible interpretation to describe deactivation of the sub-band gap absorptance would be that the sulfur dopants are initially in an optically-active chemical state and thermal annealing transforms them into a second, optically-inactive chemical state. However, such a simple two-state interpretation is not sufficient to explain the nonmonotonic trend of $n_{\mathrm{s}}$. The simplest interpretation that can account for all three data sets in Fig. 5 appears to require three states:

1. Optically active, causes carrier scattering that limits $\mu_{e}$, and is characterized by an ionization energy $E_{1}$.

2. Optically inactive, causes similar carrier scattering to State 1 , and is characterized by a smaller ionization energy $E_{2}<E_{1}$.

3. Optically inactive, causes limited carrier scattering, is not mobile by diffusion, and produces few carriers.

Upon annealing, the sulfur chemical state may evolve from State $1 \rightarrow$ State $2 \rightarrow$ State 3 . We can not conclusively identify the microscopic structure associated with each of these states using the data at hand, but we provide one possible assignment of the sulfur chemical structure for the three states listed above based on well-established properties of sulfur dopants in silicon at dilute concentrations.

At dilute concentrations, isolated sulfur impurities in silicon produce deep-level states. Single $\mathrm{S}$ atoms at substitutional lattice positions $\left(S_{S}\right)$, and two $S$ atoms on two adjacent lattice sites (substitutional dimer, $\mathrm{S}_{2 \mathrm{~S}}$ ) have been associated with levels having ionization energies of $318 \mathrm{meV}$ and $188 \mathrm{meV}$ respectively. Larger sulfur complexes $\left(\mathrm{S}_{\mathrm{C}}\right)$ have been associated with shallower donor levels ranging from $82-110 \mathrm{meV} .{ }^{38} \mathrm{We}$ propose that the initial chemical state (State 1) is comprised of primarily $\mathrm{S}_{\mathrm{S}}$, and that the increase in $n_{\mathrm{s}}$ upon annealing (shown in Fig. 5(c)) is due to the aggregation of $\mathrm{S}_{\mathrm{S}}$ into larger complexes (transformation from State $1 \rightarrow$ State 2 listed above). Theoretical calculations of the formation energy for different sulfur defects in silicon suggest that such a dimerization $\left(2 S_{S} \rightarrow S_{2 S}\right)$ is energetically favorable. ${ }^{39,40}$ As the sulfur dopants aggregate into larger clusters, the total number of defects decreases, but the ionization energy of the defects also decreases. Since $n_{\mathrm{s}}$ depends exponentially on the defect ionization energy but only proportionally on the number of defects, this transformation could explain the initial observed increase in $n_{\mathrm{s}}$ (Fig. 5(c)).

The formation of multi-atom complexes with ionization energies shallower than their single-atom components has been reported previously for other deep-level chalcogen impurities annealed in silicon. ${ }^{41,42}$ In contrast, and as expected, no such increase in $n_{\mathrm{s}}$ has been observed upon annealing silicon hyperdoped with shallow dopant impurities (B, P, As, Sb). ${ }^{4,15,16,20,21}$ The ionization energies of isolated impurities for these shallow dopants are so small that they are essentially all ionized at room temperature, so producing clusters with smaller ionization energies would not result in an increase in $n_{\mathrm{s}}$. Thus, the deep-level defects studied here provide a unique opportunity for insight to the formation of impurity complexes in supersaturated material.

The third state (State 3 listed above) may be an even larger multi-atom sulfur complex, some kind of sulfurvacancy complex, or even a sulfur precipitate (though, as mentioned above in reference to Fig. 2(b), such a precipitate would need to be very small and/or latticed matched with $\mathrm{Si})$. If this third state produces few carriers, it would explain 
the final decrease and plateau observed in $n_{\mathrm{s}}$ for $\tilde{Q}>10^{4}$. Additionally, if State 3 is a neutral complex, then, due to its neutrality, it would cause less carrier scattering than the ionized impurities in State 1 and State 2. This reduced scattering would explain why $\mu_{e}$ remains essentially unchanged during the first phase of the evolution $\left(\tilde{Q}<10^{4}\right.$, increasing $\left.n_{\mathrm{s}}\right)$, and why it increases significantly thereafter.

This three state interpretation is a potential explanation for the diffusional decomposition of sulfur-hyperdoped silicon with annealing: the supersaturation drives a local change in the chemical composition as sulfur atoms initially in solution diffuse together to form pairs and then larger clusters. It is not possible to determine what fraction of the sulfur dopant undergoes this transformation, in part because experimental evidence cannot rule out the existence of pairs and larger clusters of the sulfur dopant in the initial metastable solution. The sulfur concentration in the hyperdoped layer is approximately $3 \times 10^{20} \mathrm{~cm}^{-3}$. This concentration corresponds to average dopant separation of about $1.5 \mathrm{~nm}$. Diffusion on this length scale can not be resolved in the SIMS data shown in Fig. 2(a). If the third and final state is a slightly larger complex that is stable against further diffusion, this would explain why long-range mass transport is not observed in the fully deactivated, annealed sample.

The above interpretation provides just one potential explanation of the underlying mechanism in the deactivation of sulfur-hyperdoped silicon that is consistent with the data presented here. One alternative explanation for the final decrease in $n_{\mathrm{s}}$ and the corresponding increase in $\mu_{e}$ is that upon annealing, the measured, depth-averaged values represent an increasing contribution from the deepest, tail portion of the hyperdoped layer. This region has a lower dopant concentration, and therefore should have a lower carrier concentration and a higher mobility due to reduced impurity scattering. Further experimental measurements and analysis are needed to precisely identify the sulfur chemical states that evolve with annealing and to test the three-state mechanism proposed above. However, if, similar to oxygen, sulfur dopants evolve into a chain-like structure upon annealing, it may be difficult to distinguish between States 2 and 3 using a local probe technique (e.g., extended X-ray absorption fine structure, EXAFS), because each $\mathrm{S}$ atom shares a similar nearestneighbor environment regardless of the chain length. ${ }^{43}$

\section{CONCLUSIONS}

We examined the deactivation of single-crystal sulfurhyperdoped silicon under thermal annealing through measurements of the optical and electronic properties after a variety of different anneal conditions (anneal temperature and duration). Due to the single-crystal nature of the material considered here, we can rule out deactivation by heterogeneous nucleation at grain boundaries, which has been proposed as the deactivation mechanism in chalcogen-hyperdoped polycrystalline silicon. ${ }^{22}$ Additionally, the SIMS measurements presented here rule out long-range diffusion of the sulfur dopants as the deactivation mechanism.

We found that the deactivation of the sub-band gap optical absorptance, and the evolution of the electronic mobility and carrier concentration are all well described by a single apparent activation energy, $E_{A}=1.7 \pm 0.1 \mathrm{eV}$. We used this energy to calculate the extent of reaction for all of the temperature-duration anneal conditions, and we found that $\bar{A}, n_{\mathrm{s}}$, and $\mu_{e}$ all collapse onto distinct curves as functions of the extent of reaction: $\bar{A}$ decreases monotonically; $n_{\mathrm{s}}$ first increases by about $60 \%$ and then decreases to about $10 \%$ of its initial value; and $\mu_{e}$ is initially flat and subsequently increases monotonically by up to a factor of 6 .

We proposed that deactivation occurs as a result of short-range thermally activated atomic movements of the sulfur dopants, and we offered a possible mechanistic interpretation in which the sulfur dopants evolve through three chemical states during deactivation. We assigned the initial, optically active state to isolated substitutional sulfur impurities, and the second, optically inactive state to sulfur dimer impurities. The transformation from single substitutional impurities to dimers could explain the observed decrease in $\bar{A}$ due to the reduced number of optically active defects, the observed increase in $n_{\mathrm{s}}$ due to the lower ionization energy of sulfur dimers, and the observed unchanged $\mu_{e}$ due to dominant ionized impurity scattering in both cases. We assigned the third state to a larger, neutral sulfur complex, and transformation to this final state could explain the eventual decrease in $n_{\mathrm{s}}$ and the rise in $\mu_{e}$ due to the neutrality of the defect. This interpretation is just one possible assignment of the chemical states that is consistent with the experimental data presented here.

\section{ACKNOWLEDGMENTS}

We thank Filippo Fabbri, Matthew J. Smith, Mark T. Winkler, Elif Ertekin, and David Bell for useful discussions. Research at MIT was supported by in part by the MITKFUPM Center for Clean Water and Energy; the National Science Foundation grant for Energy, Power, and Adaptive Systems under Contract No. ECCS-1102050; and the National Science Foundation (NSF) and the Department of Energy (DOE) under NSF Contract No. EEC-1041895. Research at Harvard was supported by the U.S. ArmyARDEC under Contract No. W15QKN-07-P-0092 and the U.S. Army Research Office under Contract No. W911NF12-1-0196. J.J.K. was supported by NSERC (the Natural Sciences and Engineering Research Council of Canada). This work was performed in part at the Center for Nanoscale Systems (CNS), a member of the National Nanotechnology Infrastructure Network (NNIN), which is supported by the National Science Foundation under NSF Award No. ECS-0335765.

${ }^{1}$ C. W. White, S. R. Wilson, B. R. Appleton, and F. W. Young, J. Appl. Phys. 51, 738 (1980).

${ }^{2}$ N. Natsuaki, M. Tamura, and T. Tokuyama, J. Appl. Phys. 51, 3373 (1980).

${ }^{3}$ J. M. Poate and W. L. Brown, Phys. Today 35(6), 24 (1982).

${ }^{4}$ K. Itoh, Y. Sasaki, T. Mitsuishi, M. Miyao, and M. Tamura, Jpn. J. Appl. Phys., Part 2 21, L245 (1982).

${ }^{5}$ A. N. Larsen, F. T. Pedersen, G. Weyer, R. Galloni, R. Rizzoli, and A. Armigliato, J. Appl. Phys. 59, 1908 (1986). 
${ }^{6}$ A. J. Said, D. Recht, J. T. Sullivan, J. M. Warrender, T. Buonassisi, P. D. Persans, and M. J. Aziz, Appl. Phys. Lett. 99, 073503 (2011).

${ }^{7}$ E. Garcia-Hemme, R. Garcia-Hernansanz, J. Olea, D. Pastor, A. Del Prado, I. Mártil, and G. Gonzalez-Diaz, Appl. Phys. Lett. 101, 192101 (2012).

${ }^{8}$ J. T. Sullivan, C. B. Simmons, J. J. Krich, A. J. Akey, D. Recht, M. J. Aziz, and T. Buonassisi, J. Appl. Phys. 114, 103701 (2013).

${ }^{9}$ D. Recht, M. J. Smith, S. Charnvanichborikarn, J. T. Sullivan, M. T. Winkler, J. M. Warrender, T. Buonassisi, J. S. Williams, S. Gradecak, and M. J. Aziz, J. Appl. Phys. 114, 124903 (2013).

${ }^{10}$ T. G. Kim, J. M. Warrender, and M. J. Aziz, Appl. Phys. Lett. 88, 241902 (2006).

${ }^{11}$ B. P. Bob, A. Kohno, S. Charnvanichborikarn, J. M. Warrender, I. Umezu, M. Tabbal, J. S. Williams, and M. J. Aziz, J. Appl. Phys. 107, 123506 (2010).

${ }^{12}$ I. Umezu, J. M. Warrender, S. Charnvanichborikarn, A. Kohno, J. S. Williams, M. Tabbal, D. G. Papazoglou, X.-C. Zhang, and M. J. Aziz, J. Appl. Phys. 113, 213501 (2013).

${ }^{13}$ J. P. Mailoa, A. J. Akey, C. B. Simmons, D. Hutchinson, J. Mathews, J. T. Sullivan, D. Recht, M. T. Winkler, J. S. Williams, J. M. Warrender, P. D. Persans, M. J. Aziz, and T. Buonassisi, "Room-temperature sub-band gap optoelectronic response of hyperdoped silicon," Nat. Commun. (published online).

${ }^{14}$ S. R. Wilson, W. M. Paulson, R. B. Gregory, G. Tam, C. W. White, B. R. Appleton, A. K. Rai, and P. P. Pronko, J. Appl. Phys. 54, 5004 (1983).

${ }^{15}$ A. Parisini, A. Bourret, A. Armigliato, M. Servidori, S. Solmi, R. Fabbri, J. Regnard, and J. Allain, J. Appl. Phys. 67, 2320 (1990).

${ }^{16}$ S. Solmi, E. Landi, and F. Baruffaldi, J. Appl. Phys. 68, 3250 (1990).

${ }^{17}$ J. Allain, J. Regnard, A. Bourret, A. Parisini, A. Armigliato, G. Tourillon, and S. Pizzini, Phys. Rev. B 46, 9434 (1992).

${ }^{18}$ P. Rousseau, P. Griffin, W. Fang, and J. Plummer, J. Appl. Phys. 84, 3593 (1998).

${ }^{19}$ W. Luo, S. Yang, P. Clancy, and M. O. Thompson, J. Appl. Phys. 90, 2262 (2001).

${ }^{20}$ Y. Takamura, P. Griffin, and J. Plummer, J. Appl. Phys. 92, 235 (2002).

${ }^{21}$ L. Romano, A. M. Piro, V. Privitera, E. Rimini, G. Fortunato, B. G. Svensson, M. Foad, and M. G. Grimaldi, Nucl. Instrum. Methods Phys. Res. B 253, 50 (2006).

${ }^{22}$ B. R. Tull, M. T. Winkler, and E. Mazur, Appl. Phys. A 96, 327 (2009).
${ }^{23}$ J. T. Sullivan, R. G. Wilks, M. T. Winkler, L. Weinhardt, D. Recht, A. J. Said, B. K. Newman, Y. Zhang, M. Blum, S. Krause, W. L. Yang, C. Heske, M. J. Aziz, M. Baer, and T. Buonassisi, Appl. Phys. Lett. 99, 142102 (2011).

${ }^{24}$ C. H. Crouch, J. E. Carey, M. Shen, E. Mazur, and F. Y. Génin, Appl. Phys. A 79, 1635 (2004).

${ }^{25}$ M. Tabbal, T. Kim, J. M. Warrender, M. J. Aziz, B. L. Cardozo, and R. S. Goldman, J. Vac. Sci. Technol. B 25, 1847 (2007).

${ }^{26}$ M. T. Winkler, D. Recht, M.-J. Sher, A. J. Said, E. Mazur, and M. J. Aziz, Phys. Rev. Lett. 106, 178701 (2011).

${ }^{27}$ S. H. Pan, D. Recht, S. Charnvanichborikarn, J. S. Williams, and M. J. Aziz, Appl. Phys. Lett. 98, 121913 (2011).

${ }^{28}$ J. Vanhellemont, O. De Gryse, and P. Clauws, Appl. Phys. Lett. 86, 221903 (2005).

${ }^{29}$ R. Baron, J. Appl. Phys. 40, 3702 (1969).

${ }^{30}$ D. W. Koon, Rev. Sci. Instrum. 60, 271 (1989).

${ }^{31}$ D. A. Porter and K. E. Easterling, Phase Transformations in Metals and Alloys, 2nd ed. (CRC Press, 1992).

${ }^{32} \mathrm{~J}$. W. Christian, The Theory of Transformations in Metals and Alloys, 3rd ed. (Pergamon, 2002).

${ }^{33}$ E. J. Mittemeijer, Fundamentals of Materials Science: The Microstructure-Property Relationship Using Metals as Model Systems (Springer, 2010).

${ }^{34}$ A. Fiory and K. Bourdelle, Appl. Phys. Lett. 74, 2658 (1999).

${ }^{35}$ E. Ertekin, M. T. Winkler, D. Recht, A. J. Said, M. J. Aziz, T. Buonassisi, and J. C. Grossman, Phys. Rev. Lett. 108, 026401 (2012).

${ }^{36}$ F. Rollert, N. A. Stolwijk, and H. Mehrer, Appl. Phys. Lett. 63, 506 (1993).

${ }^{37}$ N. A. Stolwijk, D. Grünebaum, and M. Perret, Mater. Sci. Forum 38-41, 701 (1989).

${ }^{38}$ E. Janzen, R. Stedman, G. Grossmann, and H. G. Grimmeiss, Phys. Rev. B 29, 1907 (1984).

${ }^{39}$ A. Taskin, Semiconductors 36, 1083 (2002).

${ }^{40}$ Y. Mo, M. Bazant, and E. Kaxiras, Phys. Rev. B 70, 205210 (2004).

${ }^{41}$ A. Taskin and E. Tishkovskii, Semiconductors 32, 1162 (1998).

${ }^{42}$ A. Taskin and E. Tishkovskii, Semiconductors 36, 605 (2002).

${ }^{43}$ B. K. Newman, E. Ertekin, J. T. Sullivan, M. T. Winkler, M. A. Marcus, S. C. Fakra, M-J. Sher, E. Mazur, J. C. Grossman, and T. Buonassisi, J. Appl. Phys. 114, 133507 (2013). 